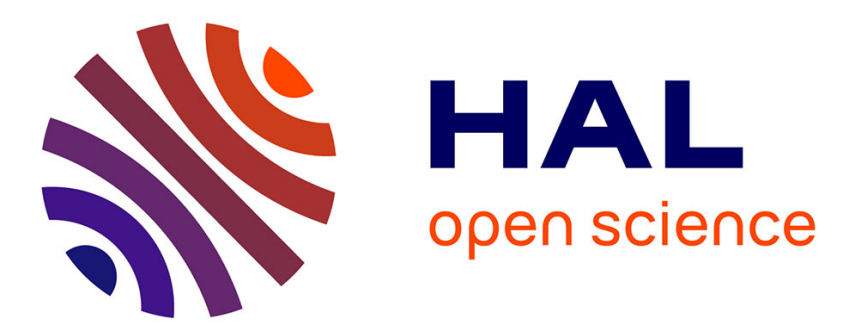

\title{
Breaking the encryption scheme of the Moscow Internet voting system
}

Pierrick Gaudry, Alexander Golovnev

\section{To cite this version:}

Pierrick Gaudry, Alexander Golovnev. Breaking the encryption scheme of the Moscow Internet voting system. Financial Cryptography and Data Security, 2020, Kota Kinabalu, Malaysia. pp.32-49, 10.1007/978-3-030-51280-4_3. hal-02266264v2

\section{HAL Id: hal-02266264 \\ https://hal.inria.fr/hal-02266264v2}

Submitted on 13 Nov 2019

HAL is a multi-disciplinary open access archive for the deposit and dissemination of scientific research documents, whether they are published or not. The documents may come from teaching and research institutions in France or abroad, or from public or private research centers.
L'archive ouverte pluridisciplinaire HAL, est destinée au dépôt et à la diffusion de documents scientifiques de niveau recherche, publiés ou non, émanant des établissements d'enseignement et de recherche français ou étrangers, des laboratoires publics ou privés. 


\title{
Breaking the Encryption Scheme of the Moscow Internet Voting System*
}

\author{
Pierrick Gaudry \\ CNRS, Inria, Université de Lorraine
}

\author{
Alexander Golovnev \\ Harvard University
}

\begin{abstract}
In September 2019, voters for the election at the Parliament of the city of Moscow were allowed to use an Internet voting system. The source code of it had been made available for public testing. In this paper we show two successful attacks on the encryption scheme implemented in the voting system. Both attacks were sent to the developers of the system, and both issues had been fixed after that.

The encryption used in this system is a variant of ElGamal over finite fields. In the first attack we show that the used key sizes are too small. We explain how to retrieve the private keys from the public keys in a matter of minutes with easily available resources.

When this issue had been fixed and the new system had become available for testing, we discovered that the new implementation was not semantically secure. We demonstrate how this newly found security vulnerability can be used for counting the number of votes cast for a candidate.
\end{abstract}

\section{Introduction}

Electronic voting is more and more widely used for low-stakes elections, with systems of various qualities. The situation for important politically binding elections is more contrasted. Some countries have completely banned the use of e-voting in that case (for instance, Germany in 2009, the Netherlands in 2008, or Norway [11] in 2013), while other countries use it on a regular basis or organize experiments with higher and higher stakes elections (Switzerland [16, 26, 8], Estonia [17], Canada [13]).

The term electronic voting can cover different situations, and in this work, we are interested in Internet voting, not machine-assisted voting that takes place in polling stations. This increases the difficulty to guarantee properties like authentication or coercion-resistance that are easier to obtain at a polling station, where an officer can check classical identity cards and where the voters can go to a polling booth to isolate themselves and choose freely.

But even more basic properties like vote secrecy and verifiability are not easy to obtain if one wants to keep things simple and without advanced cryptographic tools like zero-knowledge proofs, proof of equivalence of plaintexts, oblivious transfer, etc.

For high-stakes elections, a bad practice that tends to become less accepted by the population is to have some designated experts that study the security of the product, but how it really works remains secret to voters. Therefore, in more and more cases, the organization will ask for a product

\footnotetext{
${ }^{*}$ This work is a merger of the papers [9] and [12].
} 
that can be audited by independent experts, and as an incentive to have more feedback, public testing with an associated bug bounty program can be organized. For instance, this has been recently the case in Switzerland, which is a country with a long history of experiments with Internet voting. A security problem was actually discovered at this occasion [22, 15].

In Russia, September 8, 2019 was a day of local elections, where governors and representatives for local parliaments must be elected. In Moscow, at the occasion of this election for the City Parliament (Moscow Duma), it was decided to test the use of Internet voting. Voters from 3 electoral districts (among a total of 45 districts) were allowed to register for using Internet voting instead of using classical paper voting at polling stations.

The voting system used for this election was designed specifically. For lack of a proper name, we will call it the Moscow Internet voting system. Its deployment is the responsibility of a service of the City called the Department of Information Technology. In July, the system was opened for public testing.

\section{Description of the public challenge}

On July 17, 2019, some of the system's code was posted online [10], and the organizers asked the public to test several attack scenarios [24]. A bounty program of up to 2 millions rubles (approx. $\$ 30,000)$ was associated to it. We believed that the fact that most of the information is in Russian and that almost no description of the system (in any language) is available apart from the source code was a reason for having a low advertisement of this challenge at the international level, even among the e-voting community.

The system is poorly documented, but from the source code and brief descriptions of the system [20], we know that it uses the Ethereum blockchain [3] and ElGamal encryption. No advanced cryptographic tools are present in the source code (no verifiable mixnets [14], for instance, while they are quite frequent in modern systems).

In one of the attack scenarios, the organizers publish a challenge consisting of the public key and some encrypted messages. The attack was considered successful if the messages got decrypted within 12 hours (the duration of the future, real election), before the organizers reveal the private key and the original messages. All of these cryptographic challenges (keys and encrypted data) were put in the public repository of the source code, in a special sub-directory called encryption-keys.

\section{Contributions}

In this paper, we describe two attacks that we mounted on the system, following this attack scenario. The first attack uses the fact that the key sizes are so small that, with specialized software, it is possible to compute discrete logarithms and deduce the private keys in far less than the 12 hours allowed for this task. After this, the source code was modified. Our second attack is against this new version and relies on a subgroup attack that reveals one bit of information related to the original message. In an e-voting context, this can be enough to get a lot of information about the voter's choice, and indeed, in the Moscow system, the leakage was really strong. During August, several public tests were done, with volunteers, after the system was patched against our attacks. In this work, after describing our attacks, we will discuss the general protocol, which is some kind of moving target, since there is no proper specification, no clear security claims and on top of that, deep changes were made until very late before the real election. 
For this work, we used the following different sources of information about the Moscow Internet voting system:

- The public source code, of course. This includes Javascript code to be run on the client side, PHP code for the server side, and Solidity code to be run as smart contracts in an Ethereum blockchain.

- The articles published in the press, sometimes quoting the designers of the system. This includes various sources, with different opinions about the use of Internet voting in this context. We considered some of these sources as non-reliable.

- Private discussions with the designers and with journalists investigating the current situation.

In the following, we will refer to different versions of the source code. In order to make our terminology precise, we give the exact revision numbers of these versions, corresponding to git commits in the public repository [10]:

- The "original" version, i.e. the one that was published and used for the first public test: revision d70986b2c4da.

- The "modified" version, that took into account our first attack: revision 1d4f348681e9.

- The "final" version that was used for the election: revision 51aa4300aceb.

\section{Attacks on the encryption scheme}

\subsection{Attack on the original implementation}

In the original version of the source code (rev d70986b2c4da), the encryption scheme can be found in the files elGamal.js and multiLevelEncryptor . js of the smart-contracts/packages/crypto-lib/src/ subdirectory. The first file contains a textbook version of the ElGamal encryption algorithm, while the second one builds on top of it a "multilevel" variant that we are going to describe here since this is a non-standard construction.

Let us first fix the notations for the textbook ElGamal encryption. Let $G$ be a cyclic group generated by $g$ of order $q$. An ElGamal keypair is obtained by choosing a (secret) decryption key sk as a random integer in $\mathbb{Z}_{q}$, and the corresponding (public) encryption key pk is given by pk $=g^{\text {sk }}$. Let us denote by $\operatorname{Enc}_{g, \mathrm{pk}}(m)=(a, b)$ the ElGamal encryption of the message $m \in G$ with a public key pk and a generator $g$. This is a randomized encryption: an integer $r$ is picked uniformly at random in $\mathbb{Z}_{q}$, and then the encryption is obtained as

$$
\operatorname{Enc}_{g, \mathrm{pk}}(m)=(a, b)=\left(g^{r}, \mathrm{pk}^{r} \cdot m\right) .
$$

The corresponding decryption function $\operatorname{Dec}_{g, \mathrm{sk}}(a, b)$, that uses the secret key sk corresponding to pk is then given by

$$
\operatorname{Dec}_{g, \mathrm{sk}}(a, b)=b \cdot a^{-\mathrm{sk}}=m .
$$

The multilevel variant is obtained by successively applying the ElGamal encryption, with three different parameter sets, first on the message $m$, and then on the $a$-part of the successive ElGamal ciphertexts. In the Moscow system, there are 3 levels. Each level uses a group $G_{i}$ which is the 
multiplicative group of a finite field $\mathbb{F}_{p_{i}}$, where $p_{i}$ is a safe prime. An important remark, here, is that the $p_{i}$ 's being different, there is no algebraic map from one group to the other. It is necessary to lift an element of $\mathbb{F}_{p_{1}}^{*}$ to an integer in $\left[1, p_{1}-1\right]$ before mapping it to $\mathbb{F}_{p_{2}}^{*}$. This mapping will be without loss of information only if $p_{2}$ is larger than $p_{1}$; and similarly we need $p_{3}$ bigger than $p_{2}$. These conditions are indeed enforced in the source code.

Let us denote by $g_{1}, g_{2}, g_{3}$ the generators of the 3 groups $G_{1}, G_{2}, G_{3}$. There are 3 ElGamal key pairs $\left(\mathrm{sk}_{1}, \mathrm{pk}_{1}\right),\left(\mathrm{sk}_{2}, \mathrm{pk}_{2}\right),\left(\mathrm{sk}_{3}, \mathrm{pk}_{3}\right)$ used for the encryption and decryption of the ballots. In order to encrypt a message $m \in G_{1}$, we compute the following successive ElGamal encryptions:

$$
\begin{aligned}
\left(a_{1}, b_{1}\right) & :=\operatorname{Enc}_{g_{1}, \mathrm{pk}_{1}}(m) ; \quad \text { map } a_{1} \text { to } G_{2} ; \\
\left(a_{2}, b_{2}\right) & :=\operatorname{Enc}_{g_{2}, \mathrm{pk}_{2}}\left(a_{1}\right) ; \quad \text { map } a_{2} \text { to } G_{3} ; \\
\left(a_{3}, b_{3}\right) & :=\operatorname{Enc}_{g_{3}, \mathrm{pk}_{3}}\left(a_{2}\right),
\end{aligned}
$$

and then the ciphertext is the quadruple in $G_{1} \times G_{2} \times G_{3}^{2}$ given by

$$
\operatorname{MultiEnc}(m)=\left(b_{1}, b_{2}, a_{3}, b_{3}\right) \text {. }
$$

The values $a_{1}$ and $a_{2}$ are forgotten, but someone knowing the private keys $\mathrm{sk}_{1}, \mathrm{sk}_{2}$, sk $\mathrm{sk}_{3}$ corresponding to $\mathrm{pk}_{1}, \mathrm{pk}_{2}, \mathrm{pk}_{3}$, will be able to recover $m$ from the ciphertext with the following decryption procedure:

$$
\begin{aligned}
a_{2}:=\operatorname{Dec}_{g_{3}, \mathrm{sk}_{3}}\left(a_{3}, b_{3}\right) ; \quad \text { map } a_{2} \text { to } G_{2} ; \\
a_{1}:=\operatorname{Dec}_{g_{2}, \mathrm{sk}_{2}}\left(a_{2}, b_{2}\right) ; \quad \text { map } a_{1} \text { to } G_{1} ; \\
m:=\operatorname{Dec}_{g_{1}, \mathrm{sk}_{1}}\left(a_{1}, b_{1}\right) .
\end{aligned}
$$

The purpose of this multilevel encryption is not known to us. We will speculate on this in Section 3. An obvious observation, however, is that if the discrete logarithm problem is not hard in $G_{1}, G_{2}$ and $G_{3}$, then it is possible to deduce the secret keys $\mathrm{sk}_{i}$ 's from the public keys $\mathrm{pk}_{i}$ 's and an attacker can then decrypt encrypted messages as quickly as the legitimate possessor of the secret keys.

In the published source code, the primes $p_{i}$ 's have less than 256 bits. Discrete logarithms in finite fields defined by such small primes have been computed for the first time in the middle of the 90's: Weber, Denny and Zayer did a series of computation in 1995-1996, starting from 215 to 281 bits [33. At that time, the computing resources required for the computations were rather high, and solving the 3 discrete logarithm problems to get the private keys would not have been easily feasible in less than 12 hours as required by the challenge.

More than 2 decades later, computers are much faster and have much more memory. Furthermore, the Number Field Sieve algorithm [21], which is the fastest known method asymptotically was still a very new algorithm in the mid-90's, and many theoretical and practical optimizations have been developed since then [25, 18, 6, 30]. The current record is a computation modulo a 768-bit prime [19].

We have tried the following software products that contain a full implementation of discrete logarithm computations in prime fields:

\begin{tabular}{|c||c|c|c|}
\hline Software & SageMath [29] & Magma [4] & CADO-NFS [27] \\
\hline Version & 8.8 & $2.24-2$ & rev. 6b3746a2e \\
\hline
\end{tabular}

Note that Magma is proprietary software, while the others are free software.

The experiments were first made on a typical personal computer equipped with a 4-core Intel i5-4590 processor at $3.3 \mathrm{GHz}$ and $16 \mathrm{~GB}$ of RAM. It is running a standard Debian distribution. 
SageMath uses GP/Pari [28] internally for computing discrete logarithms. On this machine, the computation took more than 12 hours, and actually we had to stop it after 4 days while it was still running. According to GP/Pari documentation, the algorithm used is a linear sieve index calculus method. As for Magma, the handbook tells us that depending on arithmetic properties of the prime, the algorithm used can be the Gaussian integer sieve or a fallback linear sieve. The prime we tested was compatible with the Gaussian integer sieve. But during the linear algebra step, the memory requirement was much larger than the available $16 \mathrm{~GB}$. We started the computation again, on a 64-core server node with 192 GB of RAM. On this machine, Magma computed the discrete logarithm in a bit less than 24 hours with 130 GB of peak memory usage. It should be noted that both Magma and SageMath use only one of the available computing cores, so that there does not seem to be an easy way to go below the 12 hours limit with them, even with an access to a powerful machine.

CADO-NFS is an implementation of the Number Field Sieve for integer factorization and discrete logarithms in prime fields (and some experimental support for small degree extensions of prime fields). The last stable release 2.3.0 is two years old, so we used the development version, available on the public git repository. With CADO-NFS, on the standard personal machine, the running times to retrieve the private keys of August 18 were as follows:

\begin{tabular}{|c|c|}
\hline key number & time \\
\hline 1 & $425 \mathrm{sec}$ \\
2 & $507 \mathrm{sec}$ \\
3 & $314 \mathrm{sec}$ \\
\hline
\end{tabular}

Note that the variation in the running time from one key to the other is not unusual for computations with moderately small primes. Also, we should mention that when doing this work, we realized that the development version was not robust for numbers of this size: it sometimes failed in the final step called "individual logarithm" or "descent". The revision number we gave above corresponds to a version where we have fixed these problems, so that CADO-NFS can reliably compute discrete logarithms in finite fields of about 256 bits.

CADO-NFS does not include the "front-end computation" for the discrete logarithm: the small Pohlig-Hellman step due to the fact that the order of the generator is twice a prime must be done by hand; similarly, the base for the discrete logarithm computed by CADO-NFS is arbitrary. Therefore, in order to compute one of the $\mathrm{sk}_{i}$, the program must be run twice, once for the generator and once for the public key. Fortunately, in the Number Field Sieve algorithm, many parts of the computation can be shared between the two executions modulo the same prime (this is the basis of the LogJam attack [2]), and CADO-NFS indeed shares them automatically. The running times given above include those 2 runs for each key. For completeness and reproducibility, we provide in Appendix A a script to obtain the keys; this includes the few additional modular operations to be done apart from the calls to CADO-NFS.

Of course, for a real attack, the three private keys can be computed simultaneously on 3 machines in parallel. Indeed, the chaining involved in the multilevel ElGamal is not relevant for the keys, it occurs only during the encryption / decryption of messages.

Additionally to this immediate 3-fold parallelism for the attack, CADO-NFS also has some parallelism capabilities so that machines with more cores can reduce the time for a single key. However, there is some limit to it with the current implementation. For instance, the private key 
number 1 still required 160 seconds of wall clock time on the same 64-core machine that we used for testing Magma.

\section{$2.2 \quad$ Attack on the modified version}

After the first attack was sent to the developers of the system and made public a few days later, the public source code has been modified. The key size has been increased to 1024 bits, and the multilevel ElGamal has been removed and replaced by a single ElGamal encryption.

In the original version, the generators in all the involved groups were generators for the full multiplicative group of the finite fields, thus their orders were twice a prime numbers. This exposed the danger of leakage of one bit of information on the message, with a subgroup attack. This is an old technique [23], but there are still frequent attacks, in particular when an implementation forgets the key validation step [31. Although we did not push in this direction in the first attack, it was explicitly mentioned as a weakness. Therefore in the modified version, the generator was chosen to be a quadratic residue, thus having prime order.

We discovered however that the other parts of the implementation were not changed accordingly, so that an attack was still possible.

Let $p=2 q+1$ be the 1024-bit safe prime used to define the group, where $q$ is also a prime. Let $Q_{p}$ be the group of quadratic residues modulo $p$; it has order $\left|Q_{p}\right|=(p-1) / 2=q$. The chosen generator $g$ belongs to $Q_{p}$, and therefore, so is the public key pk, since it is computed as before as $\mathrm{pk}=g^{\mathrm{sk}}$, where sk is randomly chosen in $\mathbb{Z}_{q}$.

The problem with the modified implementation is that the message $m$ is allowed to be any integer from $[1, q-1]$ which is naturally mapped to an element of $\mathbb{F}_{p}^{*}$. For semantic security (under the Decisional Diffie-Hellman assumption), the message $m$ should instead be encoded as one of the $q$ elements of the group $Q_{p}$ generated by $g$. In the case where $m$ is not necessarily picked from the group of quadratic residues, the Decisional Diffie-Hellman assumption does not hold and indeed it is possible to build an efficient distinguisher, thus showing that the encryption scheme in the modified version is not semantically secure.

Let us make this explicit. If the message $m$ becomes a quadratic residue after being mapped to $\mathbb{F}_{p}^{*}$, then for every choice of randomness of the encryption algorithm, in the resulting ciphertext Enc $_{g, \mathrm{pk}}(m)=(a, b)$, the second component $b$ is also a quadratic residue. Indeed, if $g$ and $m$ belong to $Q_{p}$, then there exist $x$ and $y$ in $\mathbb{F}_{p}^{*}$ such that $g=x^{2}$ and $m=y^{2}$ Then

$$
b=\mathrm{pk}^{r} \cdot m=g^{r \cdot \mathrm{sk}} \cdot y^{2}=\left(x^{r \cdot \mathrm{sk}} y\right)^{2} \in Q_{p} .
$$

Similarly, if $m$ is not a quadratic residue, then $b=\mathrm{pk}^{r} \cdot m$ is not a quadratic residue either.

Testing the quadratic residuosity of $b$ can be done by computing the Legendre symbol of $b$ and $p$. Thanks to the law of quadratic reciprocity, a very efficient algorithm similar to the Euclidean algorithm is available [32]. Therefore from just the knowledge of a ciphertext, it is possible to immediately deduce if the corresponding cleartext $m$ belongs or not to $Q_{p}$. Roughly half of the messages are mapped to $Q_{p}$. Hence, one bit of information is leaked.

In order to test the validity of this attack, we checked whether the $b$-parts of the published encrypted messages belonged or not to $Q_{p}$. It turned out that exactly five out of the ten were quadratic residues modulo $p$. This shows that indeed, some of the cleartexts were in $Q_{p}$ and some were not. Details for reproducing these computations are given in Appendix B. 


\subsection{On the role of encryption in the protocol - What did we break?}

As in many e-voting protocols, the encryption scheme is used to encrypt the choice of the voter to form an encrypted ballot. From the Javascript source code (under a sub-directory called voting-form) that is supposed to be run on the voting device of the voter, we deduce that the encrypted data consists solely of this choice (with no additional nonce or meta-data). It takes the form of a 32-bit unsigned integer called "deputy id" that looks random.

The link between the deputy ids and the real names of the candidates is public, since the Javascript source code that must present the choices to the voters has to include it.

In the original version of the encryption scheme, as soon as the election starts, the 3 public keys of the multilevel ElGamal must become public, and from them, in a matter of minutes the decryption keys can be deduced. Then, this is as if the choices of the voters were in cleartext all along the process. Even if there is a strong trust assumption on the server that receives these votes, and even if it is honest and forgets the link between the voters and the ballots, there is still the issue of putting them in the blockchain for verifiability. Since the ballots are (essentially) in cleartext, the partial results become public all along the day of the election, which can have a strong influence on the result. Actually, it is illegal in Russia to announce any preliminary result while the election is still running.

Our second attack will not give a full information. Just one bit of information is leaked from an encrypted ballot, namely whether or not the chosen candidate has a deputy id which is a quadratic residue. As the deputy ids seem to be chosen at random with no specific arithmetic property, there is a one-half probability that they belong to $Q_{p}$, as for any element of $\mathbb{F}_{p}^{*}$. There could be some bias if the deputy ids had only a few bits, but with 32-bit integers, according to standard number theoretic heuristics this will not be the case. A plausible scenario for the attack is then a district where two candidates concentrate most of the votes, one of them having a deputy id in $Q_{p}$ and the other not. Then, from an encrypted ballot, by computing a Legendre symbol, one can deduce the voter's choice unless she voted for a less popular candidate.

Therefore, as for the first attack, this second attack means that vote secrecy relies on a very strong trust assumption in the voting server, and that the partial results are leaked all along the process.

At first, it seems that the designers were skeptical about the feasibility of this second attack, and they denied that it was a threat. However on August 28, 2019, they organized a last public testing, with only two deputy ids. It would have been fully vulnerable to the described attack, since one of the ids was in $Q_{p}$ and the other not. But despite the public source code was not yet modified, the (minified) Javascript served to the volunteers during the test included a patch against our second attack.

\section{Discussion}

\subsection{The role of the blockchain in the protocol}

\subsubsection{Blockchain as a distributed ledger}

In the protocol, the encrypted ballots are sent to an Ethereum blockchain and stored as transactions, one transaction per ballot. The argument for doing so is a typical one used in e-voting, namely offering the possibility for the voters to check that their vote is indeed taken into account. At the 
end of the election, again via the blockchain, the voters are also given a way to relate each encrypted ballot to the corresponding vote in cleartext. The goal is to provide the cast-as-intended property: if the voting client were to silently modify the choice of the voter, this would be detected.

In the above quick description, we implicitly assumed that once the voter has done the check that her ballot is present in the blockchain it will stay there and be counted in the tally. This also assumes that the voters are given enough information and tools to record the link between their vote and the corresponding entry in the blockchain, so that the check can be done in the few days (and maybe weeks or months) after the election.

In the Moscow election, a specific, permissioned Ethereum blockchain was used. The impossibility for the nodes running this blockchain to rewrite the history of the ledger in order to remove a ballot after the voter has checked it, relies therefore on the assumption that enough nodes are honest. Furthermore, the access to this specific blockchain was not guaranteed to stay for long, and actually was cut by the organizers quickly after the election.

Without access to the specifications of the protocol it is difficult to draw strong conclusions, but we consider that the verifiability properties were not as strong as what could be hoped for from a blockchain-based ledger.

\subsubsection{To use or not to use a smart contract for decryption}

In the original implementation, at the end of the election, the 3 private keys of the multilevel ElGamal were used to publicly decrypt all the ballots. This decryption was implemented in the Solidity programming language, to be run as part of a smart-contract by the nodes of the blockchain. The security properties that were sought by doing so are unclear. There are many ways to guarantee that a decryption has been correctly done, the most obvious in an ElGamal encryption setting is to include a simple zero-knowledge proof (as done for instance in Helios [1]).

In the version that was modified after our first attack, the protocol was changed, so that the decryption was done outside the smart-contract. The decryption results, namely the votes in clear, were uploaded to the blockchain as simple transactions with no computation. This operation occurs of course at the end of the election, in order to compute the tally. And additionally, the private key was also stored in the blockchain. This indeed allows the voters to verify that the decryption is correct.

Doing such a big change in the protocol just a couple of weeks before a real use in a real and high-stakes election is definitely not a good practice. However, again, without a proper specification, it is hard to deduce all the consequences. Did the trust assumptions change in the process? This also leaves open speculations about the possibility that programming the decryption in a smart contract was nothing but a peculiarity of the original design.

\subsubsection{Is it the origin of the small key sizes?}

The original code included many checks ensuring that the primes used to defined groups for the multilevel ElGamal encryption had a size small enough so that they would fit in 256 bits. This was taking the form of comparisons to a constant called SOLIDITY_MAX_INT defined as $2^{256}-1$. It indeed corresponds to the largest (unsigned) integer type natively supported by the Solidity programming language of the Ethereum smart contracts. A private communication with the designers confirmed that the reason for removing the ballot decryption of the smart-contract code and changing the 
protocol accordingly was due to the lack of time to implement a multi-precision library in Solidity, that became necessary after increasing the key size to 1024 bits.

Although the coincidence of the originally chosen bit size for the primes and the largest integer size natively supported in Solidity is striking, it is hard to be sure that this is the reason for the mistake. We can however speculate and consider that the purpose of the multilevel variant of ElGamal was to compensate for this admittedly small key size. Maybe the designers hoped for a much better security by using the three successive encryptions, just like Triple-DES is much stronger than DES. Unfortunately, things are quite different for asymmetric cryptography.

Another cause of using 256-bit keys could be the confusion between the security brought by elliptic curve cryptography and the one offered by using finite fields.

\subsection{What occurred on D-day}

The public source code repository was updated on September 6 (two days before the election) in order to take into account our second attack. In the final version the message $m$ to be encrypted is now squared before being passed to the ElGamal encryption, so that, indeed, the data that is encrypted is a quadratic residue.

The prime chosen to define the group is congruent to 3 modulo 4 . This has the following consequence: $(-1)$ is a quadratic non-residue in $\mathbb{F}_{p}^{*}$, and the Tonelli-Shanks modular square root algorithm [32] takes its simplest form, namely raising to the power $(p+1) / 4$.

In order to recover the original message after the decryption, this square root by modular exponentiation is performed, and the sign choice is based on the relative size of $p-m$ and $m$ as integers between 1 and $p-1$. Indeed, all the deputy identities that are encrypted as integers that are much smaller than $p$.

This is close to a fix we proposed when publishing our second attack, but instead of doing an additional exponentiation during encryption and having a cheap decryption, here the encryption is cheap and the decryption includes the additional exponentiation. This makes sense, since the decryption can be done on high-end servers, while the encryption is done on the voter's device which might be a smartphone.

Therefore, on September 8, the election took place with an encryption procedure which was not easy to break. Even though 1024 bits are not enough for even a medium-term security, it is certainly hard (not to say infeasible) to solve a discrete logarithm problem of that size in less than 12 hours of wall clock time. With the current public algorithmic knowledge (and extrapolations based on existing record computations [2, 19]), billions of computing cores would have to be mobilized and made to cooperate, which sounds unlikely, even with the resources of a major company or governmental agency.

According to the organizers, more than 10 thousands of Muscovites used the Internet voting system, in the 3 districts. In one of the districts, the difference between the first and the second candidates was less than 100 votes in total. This proves in retrospect the really high stakes of this experiment, since a risk of fraud in the system directly means a risk on the final result.

During the election, it was possible to access the blockchain data with a web interface, and the encrypted ballots were present in it. At the end of the day, the private key was also sent to the blockchain for verifiability purposes. But a few hours later, the access to the blockchain was cut. Fortunately, analysts of the Meduza online newspaper recorded everything and made the data 
available! They also used the private key to decrypt the 9810 encrypted ballots they found in the blockchain and published them. The statistics they observed from this data raises questions about the fairness of the election, but it is impossible to draw conclusions from just the published data.

This cutting of the access to the blockchain just after revealing the decryption key looks like an attempt to mitigate the risk on secret of the votes, while still having some kind of verifiability. This seems not to have been convincing: Soon after the election, the head of the Central Election Commission of the Russian Federation, Ella Pamfilova, made a public declaration ${ }^{2}$ clearly expressing concern about the results of this experiment and that in the coming years this should not be extended to the whole territory.

\subsection{On the absence of specification}

In our opinion, the main problems with the Moscow Internet voting system are:

- the absence of a public specification;

- the modifications made in a rush, just before the election.

In a clear specification, we expect to find much more details about the task of each entity playing a role in the system. From just the source code it is not always clear who is supposed to run some part of the code. What is also needed is clear statements about the security claims and the trust assumptions.

While the designers obviously had some verifiability properties in mind, hence used a blockchain, they certainly also wanted to maintain vote secrecy, as it is always a requirement in such a political context. It seems however, that vote secrecy with respect to the web server that received the (encrypted) ballots was not a goal. Furthermore, as far as we can see, coercion-resistance was not at all a concern, at least initially.

We do not claim that having coercion-resistance and secret with respect to the voting server is necessary for any voting system. But this should be clearly stated, so that the officials who validate the use of the system can take the decision, while knowing the risks.

This ideal process of having a clear specification, with well-stated trust assumptions and security claims is deeply incompatible with the way this election was organized. Indeed, while making a slight modification to a protocol to fix a problem is certainly feasible without having to do again the security analysis from scratch, the changes made by the designers just a few weeks or even a few days before the election were so important that they would have required to revise pages and pages of documentation if this documentation was public. And in fact, it seems that the decision to cut the access to the blockchain shortly after the end of the election was made as a quick response to some bad press about the risks on privacy and coercion. Somehow, they decided to reduce the verifiability to try to save other properties.

\section{Lessons learned and conclusion}

The first lesson learned from this story is, not surprisingly, that designers should be very careful when using cryptography. The authors of the Moscow system made many mistakes with the encryption

1 https://meduza.io/slides/meriya-sluchayno...chto-strannoe

2 https://www.kommersant.ru/doc/4095101 
scheme they decided to use. And in fact, even now, technically the encryption is still weak for two reasons. First, the 1024-bit key is too small for medium term security, and if the protocol changes so that vote privacy relies on it, this will not be enough. Furthermore, as far as we could see, the way the prime was chosen is not public, so that it could include a trapdoor making discrete logarithms easy to compute for the designers [7]. Second, textbook ElGamal, which is what is implemented now, is not IND-CCA2. Depending on the protocol, this might lead to minor or devastating attacks. As an example of the latter, in a protocol that would include a decryption oracle that allows to decrypt any ciphertext that is not in the ballot box (for instance, for audit purpose), it would be easy to use the homomorphic properties of ElGamal to get all the ballots decrypted.

The second lesson is that using a blockchain is not enough to guarantee full transparency. There are various notions of verifiability in the e-voting literature [5], and the designers must clearly say which property they have, under precise trust assumptions. These trust assumptions must be made even more carefully when using a permissioned blockchain, where the nodes running the blockchain are probably specifically chosen for the election, and where the access to the blockchain can be cut at any time.

Even more specific to e-voting, the Moscow system is a good example of the difficulty for an Internet voting system to make the vote secrecy rely uniquely on cutting the link between the voters and their encrypted ballots when they arrive on a server that should also authenticate the voters. What is really required is to cut the link with the vote in clear, and, for this, classical methods exist like homomorphic decryption or verifiable mixnets. In such a high-stakes election, many seemingly incompatible security properties must be satisfied (secrecy vs transparency), and advanced cryptographic tools are almost impossible to avoid.

Finally, as a conclusion, although our attacks led to the system using a better encryption scheme, it is clear that the system as a whole is still far from being perfect. We consider it likely that if the specification were becoming public in the future, other attacks would be revealed. Therefore, we believe that the main impact of our work was to draw the attention to the system as something that was maybe not as secure as what was claimed. The bad publicity in the press hopefully influenced some potential voters who decided not to take the risk of using this still really problematic system and went for paper ballots instead.

\section{Acknowledgements}

Thanks to Iuliia Krivonosova and Robert Krimmer, for sharing some information about the Moscow Internet voting. In particular Iuliia's blog post [20] was quite useful. We also thank Noah StephensDavidowitz for his comments on an earlier version of this note. We thank Mikhail Zelenskiy and Denis Dmitriev for sharing some data and information about the voting scheme.

\section{References}

[1] Ben Adida, Olivier De Marneffe, Olivier Pereira, and Jean-Jacques Quisquater. Electing a university president using open-audit voting: Analysis of real-world use of Helios. In Proceedings of the 2009 Conference on Electronic Voting Technology/Workshop on Trustworthy Elections, EVT/WOTE'09, pages 10-10. USENIX, 2009. 
[2] David Adrian, Karthikeyan Bhargavan, Zakir Durumeric, Pierrick Gaudry, Matthew Green, J. Alex Halderman, Nadia Heninger, Drew Springall, Emmanuel Thomé, Luke Valenta, Benjamin VanderSloot, Eric Wustrow, Santiago Zanella Béguelin, and Paul Zimmermann. Imperfect forward secrecy: How Diffie-Hellman fails in practice. In ACM CCS 15: 22nd Conference on Computer and Communications Security, pages 5-17. ACM Press, 2015.

[3] Vitalik Buterin. Ethereum white paper, 2013. GitHub repository: https://github.com/ ethereum/wiki/wiki/White-Paper.

[4] John Cannon, Wieb Bosma, Claus Fieker, and Allan Steel. Handbook of MAGMA functions, 2006. http://magma.maths.usyd.edu.au/magma/handbook/

[5] Véronique Cortier, David Galindo, Ralf Küsters, Johannes Mueller, and Tomasz Truderung. SoK: Verifiability notions for e-voting protocols. In IEEE Symposium on Security and Privacy (SEP 2016), pages 779-798. IEEE, 2016.

[6] Jens Franke and Thorsten Kleinjung. Continued fractions and lattice sieving. In Special-Purpose Hardware for Attacking Cryptographic Systems-SHARCS, 2005. 40 pages.

[7] Joshua Fried, Pierrick Gaudry, Nadia Heninger, and Emmanuel Thomé. A kilobit hidden SNFS discrete logarithm computation. In EUROCRYPT 2017, volume 10210 of LNCS, pages 202-231. Springer, 2017.

[8] David Galindo, Sandra Guasch, and Jordi Puiggali. 2015 Neuchâtel's cast-as-intended verification mechanism. In 5th International Conference on E-Voting and Identity, (VoteID'15), pages 3-18, 2015.

[9] Pierrick Gaudry. Breaking the encryption scheme of the Moscow internet voting system. arXiv:1908.05127, 2019.

[10] Public source code of the Moscow internet voting system. https://github.com/ moscow-technologies/blockchain-voting, 2019.

[11] Kristian Gjøsteen. The Norwegian Internet voting protocol. In E-Voting and Identity, volume 7187 of LNCS, pages 1-18. Springer, 2012.

[12] Alexander Golovnev. An attack on the the encryption scheme of the Moscow internet voting system. arXiv:1908.09170, 2019.

[13] Nicole J Goodman. Internet voting in a local election in Canada. In The Internet and Democracy in Global Perspective, pages 7-24. Springer, 2014.

[14] Jens Groth. A verifiable secret shuffle of homomorphic encryptions. Journal of Cryptology, 23(4):546-579, 2010.

[15] Rolf Haenni. Swiss Post public intrusion test - Generating random group elements, 2019. Preprint: https://e-voting.bfh.ch/publications/2019/.

[16] Rolf Haenni, Reto E. Koenig, Philipp Locher, and Eric Dubuis. CHVote system specification. Cryptology ePrint Archive, Report 2017/325, 2017. https://eprint.iacr.org/2017/325. 
[17] Sven Heiberg and Jan Willemson. Verifiable internet voting in Estonia. In 2014 6th International Conference on Electronic Voting: Verifying the Vote (EVOTE), pages 1-8. IEEE, 2014.

[18] A. Joux and R. Lercier. Improvements to the general number field sieve for discrete logarithms in prime fields. A comparison with the gaussian integer method. Mathematics of Computation, 72(242):953-967, 2003.

[19] Thorsten Kleinjung, Claus Diem, Arjen K Lenstra, Christine Priplata, and Colin Stahlke. Computation of a 768-bit prime field discrete logarithm. In EUROCRYPT 2017, volume 10210 of $L N C S$, pages 185-201. Springer, 2017.

[20] Julia Krivonosova. Internet voting in Russia: how? https://medium.com/ @juliakrivonosova/internet-voting-in-russia-how-9382db4da71f, 2019.

[21] A. K. Lenstra and H. W. Lenstra, Jr., editors. The development of the number field sieve, volume 1554 of Lecture Notes in Math. Springer-Verlag, 1993.

[22] Sarah Jamie Lewis, Olivier Pereira, and Vanessa Teague. Trapdoor commitments in the SwissPost e-voting shuffle proof, 2019. Blog note: https://people.eng.unimelb.edu.au/ vjteague/SwissVote.html.

[23] Chae Hoon Lim and Pil Joong Lee. A key recovery attack on discrete log-based schemes using a prime order subgroup. In CRYPTO'97, volume 1294 of LNCS, pages 249-263. Springer, 1997.

[24] Public testing of the Internet voting system. https://www.mos.ru/upload/documents/files/ 5381/Formal_Offer.pdf, 2019. In Russian.

[25] O. Schirokauer. Discrete logarithms and local units. Philos. Trans. Roy. Soc. London Ser. A, 345(1676):409-423, 1993.

[26] Scytl. Swiss On-line Voting Protocol, 2016. Manuscript.

[27] The CADO-NFS Development Team. CADO-NFS, an implementation of the number field sieve algorithm, 2019. Development version fdae0f9f382c, available from http://cado-nfs.gforge. inria.fr/.

[28] The PARI Group. PARI/GP version 2.11.0, 2018. available from http://pari.math. u-bordeaux.fr/.

[29] The Sage Developers. Sagemath, the Sage Mathematics Software System (Version 8.8), 2019. https://www.sagemath.org.

[30] E. Thomé. Subquadratic computation of vector generating polynomials and improvement of the block Wiedemann algorithm. Journal of Symbolic Computation, 33(5):757-775, 2002.

[31] Luke Valenta, David Adrian, Antonio Sanso, Shaanan Cohney, Joshua Fried, Marcella Hastings, J Alex Halderman, and Nadia Heninger. Measuring small subgroup attacks against DiffieHellman. In NDSS, 2017. 
[32] Joachim Von Zur Gathen and Jürgen Gerhard. Modern computer algebra. Cambridge university press, 2013. Third edition.

[33] Damian Weber, Thomas Denny, and Joerg Zayer. Discrete logarithms mod p: 215, 248 and 281 bit computation announcements on the NMBRTHRY mailing list, 1995. https://listserv. nodak. edu/cgi-bin/wa. exe?A0=NMBRTHRY 


\section{A A shell script for the first attack}

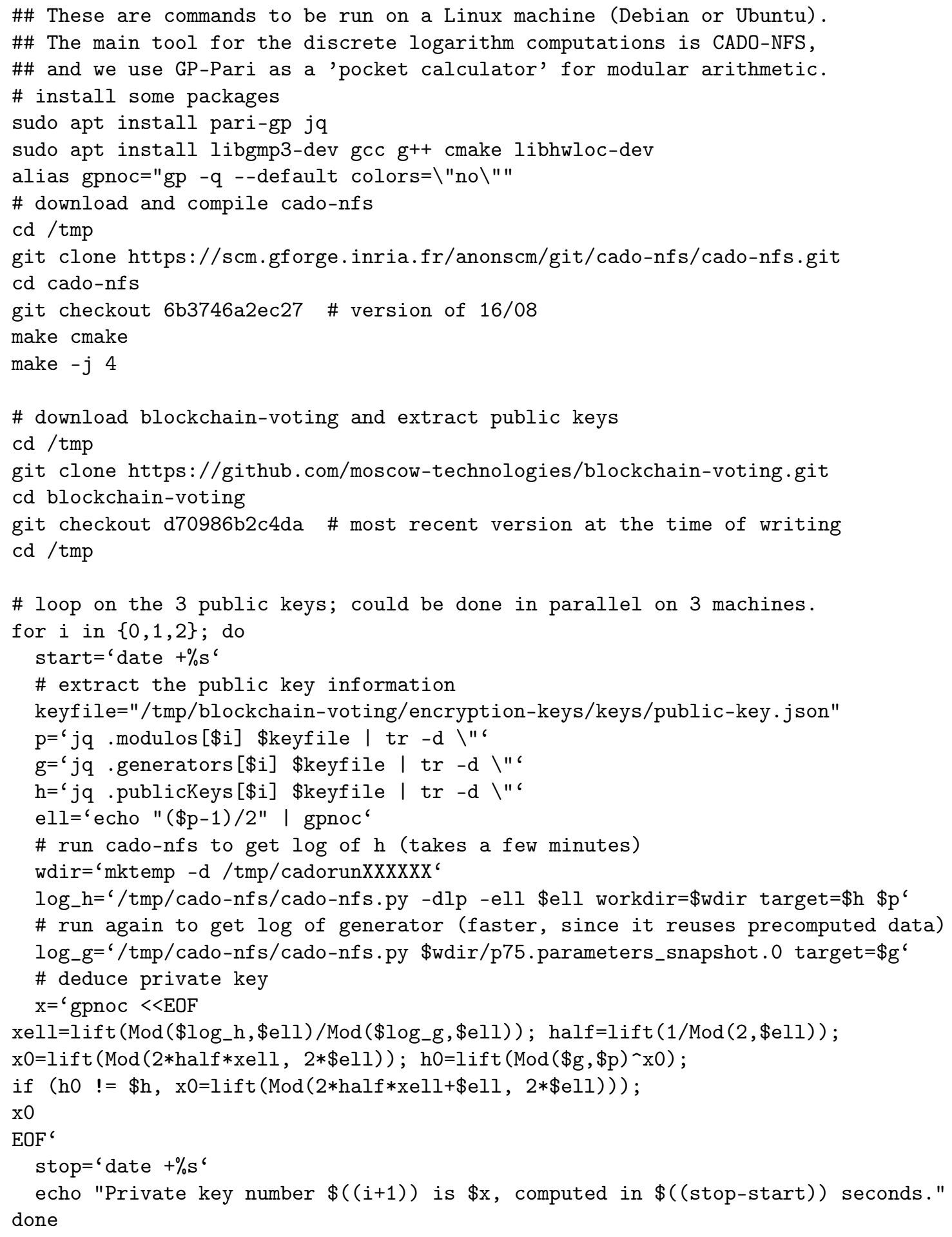




\section{B Encrypted messages are not quadratic residues}

In this Appendix we use the provided public key and encrypted messages in the modified version of the public repository [10] (revision 1d4f348681e9) to show that not all messages are quadratic residues in $\mathbb{F}_{p}^{*}$. Here $b$ is the set of the second components of the encrypted messages (that is, each element of the set is $\mathrm{pk}^{r} \cdot m$ where $m$ is some plain message, pk is the public key, and $r$ is a random number). The following Python code shows that only five out of ten elements are quadratic residues. For simplicity, we compute the Legendre symbol by modular exponentiation: the quadratic residues give 1 when raised to the power $(p-1) / 2$.

$\mathrm{p}=$ 10062759081450625618037903678618826196600591242500860802791085970455088 29615914188038720723057459046019130152450978128758867982127126946624453 23678201384359740027439588690880234391145675099291004487668846511981135 30933109486902142540395785614572268133031351548262091859360232929939444 1379077427748866822254003

$q=(p-1) / / 2$

$\mathrm{b}=[$

86911001506497462251782638567319361833688978813664946437333829354738909 40443974481927929263283486987233406326466505025027434679060583881689706 23263052860581382950559847777412555501704989450676046755496358356631412 74356550963994173797345489306417174072514309856175754908122436241421564 859178326320313204945649 , 32994578715846315625334282465389128113015193084444994471583135772127926 44951892161427453570566766298979864185170520616403124797427010730707520 16109483404053598174999416617877699551805519137361275465665467691230764 44375224889357541488942667685714188203805416972085863674686599803137288 027861639262227344813980 , 25605451399106620676652873102021964641362454624148409311459772958496440 67016843578315908545184077772794593830979151616819810966255709567920814 13077819709806694723689969137957383923170349530451483441188337477065322 87151838997509598299206147956479381022563215978764100195629663712388182 647511089787862332483202 , 30936197551567269685847042352240834287171756541862382295858852516666762 11755805979729879023007285286880732674891989007741022633330800550368742 56346941237089009381794632389798562078456796442958644789501357076108208 77962547470703268773776147336174270678101221755152924933175072952910690 305403946708512011344065 , 90189227659365697355063500941760536836478537551461759945631823319091683 13130539947043416222984580270526152593756457555485599018740243229324226 84960561239260442729637671756134870576696053584273031857981168518983390 53864084929055706240055307151918736952456608210700937953363208336695605 308414504363789714782355 , 91764714915834445310265717136195446845915020510854708634828807741642908 65088805234016509342009913809428795919722926613847539079055997816788187 99170526245002211336442034207826902363786376681934271623388852857592304 13278401533846260888398253877915981254520562872698617685705979612448346 470413913994244174120780 , 53180133541691920877303393106622876213880557470163604793597655634027675 


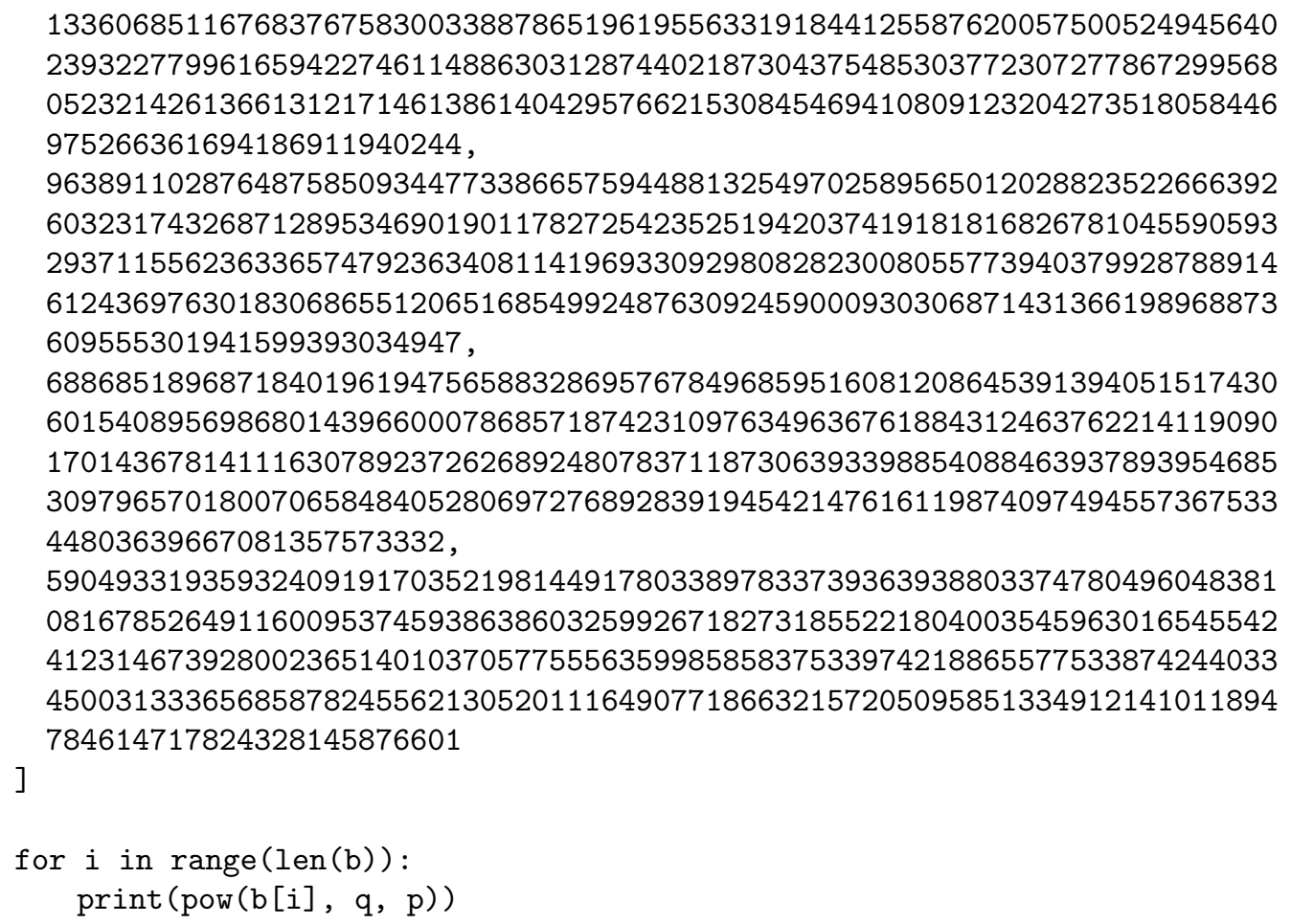

\section{Parameters from the public testing held on August 28}

In this Appendix we show the encryption parameters used for the last public testing held on August 28,2019 . These were not included in the GitHub public repository but were extracted from the Javascript code sent to the voters. There were two candidates, one option corresponded to a quadratic residue, while the other one corresponded to a quadratic non-residue. Therefore, the second attack described in Section 2.2 would have decoded all votes.

$\mathrm{p}=$

12270848251665690851841155105748670756648053237913900516699359405362771

39717263095726449865110213728719981659033550058365258369834144969686617 29191112587333253191262755602784412922675331893614019119979108938727080 35007007749458130783976450013979645236359373116042676595576310035726012 4300619948890487736216143

$\mathrm{q}=(\mathrm{p}-1) / / 2$

$\mathrm{m} 1=3247602110$

$\mathrm{m} 2=667396531$

print (pow (m1, q, p))

print (pow (m2, q, p)) 